\title{
A New Model for Calculating the Ground and Excited States Masses Spectra of Doubly Heavy $\Xi$ Baryons
}

\author{
Neda Mohajery, ${ }^{1}$ Nasrin Salehi $\left(\mathbb{D},{ }^{1}\right.$ and Hassan Hassanabadi ${ }^{2}$ \\ ${ }^{1}$ Department of Basic Sciences, Shahrood Branch, Islamic Azad University, Shahrood, Iran \\ ${ }^{2}$ Faculty of Physics, Shahrood University of Technology, Shahrood, Iran \\ Correspondence should be addressed to Nasrin Salehi; salehi@shahroodut.ac.ir
}

Received 9 March 2018; Revised 31 May 2018; Accepted 11 June 2018; Published 26 July 2018

Academic Editor: Chun-Sheng Jia

Copyright (c) 2018 Neda Mohajery et al. This is an open access article distributed under the Creative Commons Attribution License, which permits unrestricted use, distribution, and reproduction in any medium, provided the original work is properly cited. The publication of this article was funded by $\mathrm{SCOAP}^{3}$.

\begin{abstract}
Since the doubly heavy baryons masses are experimentally unknown (except $\Xi_{c c}^{+}$and $\Xi_{c c}^{++}$), we present the ground state masses and the positive and negative parity excited state masses of doubly heavy $\Xi$ baryons. For this purpose, we have solved the sixdimensional hyperradial Schrödinger equation analytically for three particles under the hypercentral potential by using the ansatz approach. In this paper, the hypercentral potential is regarded as a combination of the color Coulomb plus linear confining term and the six-dimensional harmonic oscillator potential. We also added the first-order correction and the spin-dependent part contains three types of interaction terms (the spin-spin term, spin-orbit term, and tensor term) to the hypercentral potential. Our obtained masses for the radial excited states and orbital excited states of $\Xi_{c c d}, \Xi_{c c u}, \Xi_{b b d}, \Xi_{b b u}, \Xi_{b c d}$, and $\Xi_{b c u}$ systems are compared with other theoretical reports, which could be a beneficial tool for the interpretation of experimentally unknown doubly heavy baryons spectrum.
\end{abstract}

\section{Introduction}

The doubly heavy baryons have two heavy quarks ( $c$ and $b$ ) with a light quark ( $\mathrm{d}$ or $\mathrm{u}$ or s). The doubly heavy $\Xi$ baryons family have up or down quarks but $\Omega$ family has a light strange quark and their masses spectra have been predicted in the quark model [1]. The SELEX collaboration announced only the experimental mass for the ground state of $\Xi_{c c}^{+}$baryon and LHCb has determined the ground state of $\Xi_{c c}^{++}$baryon mass while no triply heavy baryons have been observed yet [2]. Recently experiments and theoretical outcomes have been used in studying the heavy baryons. A lot of new experimental results have been reported by various experimental facilities like CLEO, Belle, BaBar, LHCb, and so forth $[3,4]$ on ground states and many new excited states of heavy flavor baryons. Bottom baryons are investigated at LHC and Lattice QCD whereas charm baryons are announced at the $\mathrm{B}$-factories $[5,6]$. On the other hand, the theoretical works are providing new results for doubly heavy baryons like the Hamiltonian model [7], relativistic quark model [8], the chiral unitary model [9], QCD sum rule [10, 11], and many more. Single- and double- heavy baryons in the constituent quark model were studied by Yoshida et al. They used a model in which there were two exceptions, a color Coulomb term depending on quark masses and an antisymmetric L.S force. They studied the low-lying negative parity states and structures within the framework of a constituent quark model [7]. In [12], the authors calculated the masses of baryons with the quadratic mass relations for ground and orbitally excited states. Wei et al. estimated the masses of singly, doubly, and triply bottom baryons in [13]. Then they studied the linear mass relations and quadratic mass relations.

The light flavor dependence of the singly and doubly charmed states is investigated by Rubio et al. They focused on searching the masses of charmed baryons with positive and negative parity [5]. In [14], the authors used lattice QCD for baryons containing one, two, or three heavy quarks. They applied nonrelativistic QCD for the bottom quarks and relativistic heavy-quark action for the charm quarks. Padmanath et al. determined the ground and excited state spectra of doubly charmed baryons from lattice QCD with dynamical quark fields [15]. The mass of the heavy baryons with two heavy b or $\mathrm{c}$ quarks for spin $1 / 2$ in the framework of 
QCD sum rules is estimated by Aliev et al. They use the most general form of the interpolating current in its symmetric and antisymmetric forms with respect to the exchange of heavy quarks, to calculate the two point correlation functions describing the baryons under consideration [16]. The authors calculated the masses and residues of the spin $3 / 2$ doubly heavy baryons within the QCD sum rules method. In [17], Eakins et al. ignored all spin-dependent interactions and assume a flavor independent potential, working in the limit where the two heavy quarks are massive enough that their motion can be treated as essentially nonrelativistic, and QCD interactions can be well described by an adiabatic potential [18]. The three-quark problem was solved by Valcarce et al. by means of the Faddeev method in momentum space [19].

The masses of the ground and excited states of the doubly heavy baryons were calculated by Ebert et al. baryons on the basis of the quark-diquark approximation in the framework of the relativistic quark model [20]. In [21], the authors, in the model with the quark-diquark factorization of wave functions, estimated the spectroscopic characteristics of baryons containing two heavy quarks. Albertus et al. used five different quark-quark potentials that include a confining term plus Coulomb and hyperfine terms coming from one-gluon exchange. They solved the three-body problem by means of a variational ansatz made possible by heavy-quark spin symmetry constraints [22].

In this study, we have used the hypercentral constituent quark model (hCQM) with Coulombic-like term plus a linear confining term and the harmonic oscillator potential [23]. We also added the first-order correction and the spin-dependent part to the potential and calculation has been performed by solving six-dimensional hyperradial Schrödinger equations by using the ansatz method. We have obtained the mass spectra of radial excited states up to $5 \mathrm{~S}$ and orbital excited states for $1 \mathrm{P}-5 \mathrm{P}, 1 \mathrm{D}-4 \mathrm{D}$, and $1 \mathrm{~F}-2 \mathrm{~F}$ states.

This paper is organized as follows: we briefly present the hypercentral constituent quark model and introduce the interaction potentials between three quarks in doubly heavy baryons in Section 2. In Section 3, we present the exact analytical solution of the hyperradial Schrödinger equation for our proposed potential. In Section 4, our masses spectra results for ground, radial, and orbital excited states of baryon family with six members are given and compared with other predictions. We present the conclusions in Section 5.

\section{Theoretical Framework: The HCQM Model and Hypercentral Potential}

The hypercentral model has been applied to solve bound states and scattering problems in many various fields of physics. In this model, we consider baryons as three-body systems of constituent quarks. In the center of mass frame, the internal quark motion is described by the Jacobi coordinates ( $\rho$ and $\lambda$ ) [37] and the respective reduced masses are given by

$$
\begin{aligned}
& m_{\rho}=\frac{2 m_{1} m_{2}}{m_{1}+m_{2}}, \\
& m_{\lambda}=\frac{2 m_{3}\left(m_{1}^{2}+m_{2}^{2}+m_{1} m_{2}\right)}{\left(m_{1}+m_{2}\right)\left(m_{1}+m_{2}+m_{3}\right)}
\end{aligned}
$$

Here $m_{1}, m_{2}$, and $m_{3}$ are the current quark masses. In order to describe three-quark dynamics, we define hyperradius $x=$ $\sqrt{\rho^{2}+\lambda^{2}}$ and hyperangle $\xi=\arctan (\rho / \lambda)$ [38]. In present work, the confining three-body potential is regarded as a combination of three hypercentral interacting potentials. First, the six-dimensional hyper-Coulomb potential $V_{h y c}(x)=\tau / x$, which is attractive for small separations [39-41], while at large separations a hyper-linear term, $V_{\text {con }}=\beta x$, gives rise to quark confinement [42], where $\beta$ corresponds to the string tension of the confinement [43]. Third, the six-dimension harmonic oscillator potential $V_{\text {h.o. }}=p x^{2}$, which has a two-body character and turns out to be exactly hypercentral [44], where $p$ is constant. The solution of the hypercentral Schrödinger equation with Coulombic-like term plus a linear confining term potential cannot be obtained analytically [45]; therefore, Giannini et al. used the dynamic symmetry $\mathrm{O}(7)$ of the hyper-Coulomb problem to obtain the hyper-Coulomb Hamiltonian and eigenfunctions analytically and they regarded the linear term as a perturbation. Combination of the color Coulomb plus linear confining term and the six-dimensional harmonic oscillator potential has interesting properties since it can be solved analytically, with a good correspondence to physical results. The first-order correction $V^{(1)}(x)$ can be written as [44-47]

$$
V^{1}(x)=-C_{F} C_{A} \frac{\alpha_{S}^{2}}{4 x^{2}}
$$

The parameters $C_{F}=2 / 3$ and $C_{A}=3$ are the Casimir charges of the fundamental and adjoint representation. The hyperCoulomb strength $\tau=-(2 / 3) \alpha_{S}, 2 / 3$ is the color factor for the baryon. $\alpha_{s}$ is the strong running coupling constant, which is written as

$$
\begin{aligned}
& \alpha_{S} \\
& =\frac{\alpha_{S}\left(\mu_{0}\right)}{1+\left(\left(33-2 n_{f}\right) / 12 \pi\right) \alpha_{S}\left(\mu_{0}\right) \ln \left(\left(m_{1}+m_{2}+m_{3}\right) / \mu_{0}\right)}
\end{aligned}
$$

The spin-dependent part $V_{S D}(x)$ is given as

$$
\begin{aligned}
V_{S D}(x)= & V_{S S}(x)\left(\vec{S}_{\rho} \cdot \vec{S}_{\lambda}\right)+V_{\gamma S}(x)(\vec{\gamma} \cdot \vec{S}) \\
& +V_{T}(x)\left[S^{2}-\frac{3(\vec{S} \cdot \vec{x})(\vec{S} \cdot \vec{x})}{x^{2}}\right]
\end{aligned}
$$

The spin-dependent potential, $V_{S D}(x)$, contains three types of the interaction terms [48], such as the spin-spin term $V_{S S}(x)$, the spin-orbit term $V_{\gamma S}(x)$, and tensor term $V_{T}(x)$ described as [35]. Here $S=S_{\rho}+S_{\lambda}$, where $S_{\rho}$ and $S_{\lambda}$ are the spin vectors associated with the $\rho$ and $\lambda$ variables, respectively. The coefficient of these spin-dependent terms of the above equation can be written in terms of the vector, $V_{V}(x)=\tau / x$, and scalar, $V_{S}(x)=\beta x+p x^{2}$ parts of the static potential as [38]

$$
V_{\gamma s}=\frac{1}{2 m_{\rho} m_{\lambda} x}\left(3 \frac{d V_{V}}{d x}-\frac{d V_{S}}{d x}\right)
$$


TABLE 1: The quark mass (in GeV) and the fitted values of the parameters used in our calculations.

\begin{tabular}{ccccccccc}
\hline$m_{b}$ & $m_{c}$ & $m_{d}$ & $m_{u}$ & $\alpha_{S}$ & $C_{F}$ & $C_{A}$ & $\beta$ & $\omega$ \\
\hline 4.750 & 1.348 & 0.35 & 0.34 & 0.340 & $\frac{2}{3}$ & 3 & 0.02 & $0.11 \mathrm{fm}^{-1}$ \\
\hline
\end{tabular}

TABLE 2: The outcomes ground state masses of $\Xi$ are listed with other theoretical predictions (in GeV). Standard devotion of the result is 0.350 .

\begin{tabular}{|c|c|c|c|c|c|c|}
\hline \multirow{2}{*}{$\begin{array}{l}\text { Baryon } \\
J^{P}\end{array}$} & \multicolumn{2}{|c|}{$\Xi_{c c d} / \Xi_{c c u}$} & \multicolumn{2}{|c|}{$\Xi_{b b d} / \Xi_{b b u}$} & \multicolumn{2}{|c|}{$\Xi_{b c d} / \Xi_{b c u}$} \\
\hline & $\frac{1}{2}^{+}$ & $\frac{3}{2}^{+}$ & $\frac{1}{2}^{+}$ & $\frac{3}{2}^{+}$ & $\frac{1}{2}^{+}$ & $\frac{3}{2}^{+}$ \\
\hline Our Calc & $3.522 / 3.515$ & $3.696 / 3.689$ & $9.716 / 9.711$ & 9.894 / 9.889 & $6.628 / 6.622$ & $6.688 / 6.682$ \\
\hline Ref.[1] & $3.520 / 3.511$ & 3.695 / 3.687 & $10.317 / 10.312$ & $10.340 / 10.335$ & 6.920 / 6.914 & $6.986 / 6.980$ \\
\hline Ref.[24] & 3.519 & & & & & \\
\hline Ref.[7] & 3.685 & 3.754 & 10.314 & & & \\
\hline Ref.[12, 13] & 3.520 & 3.695 & 10.199 & 10.316 & & \\
\hline Ref.[5] & 3.610 & 3.694 & & & & \\
\hline Ref.[14] & 3.610 & 3.692 & 10.143 & 10.178 & 6.943 & 6.985 \\
\hline Ref.[25] & 3.561 & 3.642 & & & & \\
\hline Ref.[17] & 3.720 & & 9.960 & & 6.720 & \\
\hline Ref.[18] & 3.687 & 3.752 & 10.322 & 10.352 & 7.014 & 7.064 \\
\hline Ref.[26] & 3.676 & 3.753 & 10.340 & 10.367 & 7.011 & 7.074 \\
\hline Ref.[27] & 3.547 & 3.719 & 10.185 & 10.216 & 6.904 & 6.936 \\
\hline Ref.[19] & 3.579 & 3.656 & 10.189 & 10.218 & & \\
\hline Ref.[20] & 3.620 & 3.727 & 10.202 & 10.237 & 6.933 & 6.980 \\
\hline Ref.[21] & 3.478 & 3.610 & 10.093 & 10.133 & 6.820 & 6.900 \\
\hline Ref.[28] & 3.627 & 3.690 & 10.162 & 10.184 & 6.914 & \\
\hline Ref.[29] & 3.519 & 3.620 & 9.800 & 9.980 & 6.650 & 6.690 \\
\hline Ref.[22] & 3.612 & 3.706 & 10.197 & 10.136 & 6.919 & 6.986 \\
\hline Ref.[30] & 3.510 & 3.548 & 10.130 & 10.144 & 6.792 & 6.827 \\
\hline Ref.[31] & 3.570 & 3.610 & 10.170 & 10.220 & & \\
\hline
\end{tabular}

$$
\begin{aligned}
V_{T}(x) & =\frac{1}{6 m_{\rho} m_{\lambda}}\left(\frac{3 d^{2} V_{V}}{d^{2} x}-\frac{1}{x} \frac{d V_{V}}{d x}\right) \\
V_{S S}(x) & =\frac{1}{3 m_{\rho} m_{\lambda}} \nabla^{2} V_{V}
\end{aligned}
$$

In our model, the hypercentral interaction potential is assumed as follows [48]:

$$
V(x)=V^{(0)}(x)+\left(\frac{1}{m_{\rho}}+\frac{1}{m_{\lambda}}\right) V^{(1)}(x)+V_{S D}(x)
$$

where $V^{(0)}(x)$ is given by

$$
\begin{aligned}
V^{(0)}(x) & =V_{\text {hyc }}(x)+V_{\text {con }}(x)+V_{\text {h.o. }}(x) \\
& =\frac{\tau}{x}+\beta x+p x^{2}
\end{aligned}
$$

The baryons masses are determined by the sum of the model quark masses plus kinetic energy, potential energy, and the spin-dependent interaction as $M_{B}=\sum m_{i}+\langle H\rangle$ [49]. First, we have solved the hyperradial Schrödinger equation exactly and find eigenvalue under the proposed potential by using the ansatz approach.

\section{The Exact Analytical Solution of the Hyperradial Schrödinger Equation under the Hypercentral Potential}

The Hamiltonian of three bodies' baryonic system in the hypercentral constituent quark model is expressed as [50]

$$
H=\frac{P_{\rho}^{2}}{2 m}+\frac{P_{\lambda}^{2}}{2 m}+V(\mathrm{x})
$$

and the hyperradial wave function $\psi_{\nu \gamma}(x)$ is determined by the hypercentral Schrödinger equation. The hyperradial Schrödinger equation corresponding to the above Hamiltonian can be written as [51]

$$
\begin{gathered}
\left(\frac{d^{2}}{d x^{2}}+\frac{5}{x} \frac{d}{d x}-\frac{\gamma(\gamma+4)}{x^{2}}\right) \psi_{\nu \gamma}(x) \\
=-2 m[E-V(x)] \psi_{\nu \gamma}(x)
\end{gathered}
$$

where $\gamma$ is the grand angular quantum number and given by $\gamma=2 n+l_{\rho}+l_{\lambda}, n=0,1, \ldots ; l_{\rho}$ and $l_{\lambda}$ are the angular momenta associated with the $\vec{\rho}$ and $\vec{\lambda}$ variable and $\nu$ denotes the number of nodes of the space three-quark wave function [36]. In (11), $m$ is the reduced mass which is defined as $m=$ 
TABLE 3: The masses of radial excited states for doubly heavy $\Xi$ baryons (in GeV). Standard devotions of the result are 0.435 and 0.434 .

\begin{tabular}{|c|c|c|c|c|c|c|c|c|c|c|c|c|}
\hline Baryon & State & $J^{P}$ & Our Calc & Our Calc & {$[1]$} & {$[1]$} & [7] & {$[26]$} & [27] & [19] & {$[20]$} & {$[18]$} \\
\hline \multirow{8}{*}{$\begin{array}{l}\Xi_{c c d} \\
\text { and } \\
\Xi_{c c u}\end{array}$} & $2 S$ & & 3.905 & 3.901 & 3.925 & 3.920 & 4.079 & 4.029 & 4.183 & 3.976 & 3.910 & 4.030 \\
\hline & $3 S$ & $1^{+}$ & 4.185 & 4.118 & 4.233 & 4.159 & 4.206 & & 4.640 & & 4.154 & \\
\hline & $4 S$ & 2 & 4.430 & 4.429 & 4.502 & 4.501 & & & & & & \\
\hline & $5 S$ & & 4.653 & 4.653 & 4.748 & 4.748 & & & & & & \\
\hline & $2 S$ & & 3.962 & 3.958 & 3.988 & 3.983 & 4.114 & 4.042 & 4.282 & 4.025 & 4.027 & 4.078 \\
\hline & $3 S$ & $3^{+}$ & 4.213 & 4.211 & 4.264 & 4.261 & 4.131 & & 4.719 & & & \\
\hline & $4 \mathrm{~S}$ & $\overline{2}$ & 4.446 & 4.445 & 4.520 & 4.519 & & & & & & \\
\hline & $5 \mathrm{~S}$ & & 4.663 & 4.663 & 4.759 & 4.759 & & & & & & \\
\hline \multirow{7}{*}{$\begin{array}{l}\Xi_{b b d} \\
\text { and } \\
\Xi_{b b u}\end{array}$} & $2 S$ & & 9.984 & 9.981 & 10.612 & 10.609 & 10.571 & 10.576 & 10.751 & 10.482 & 10.441 & 10.551 \\
\hline & $3 S$ & $1^{+}$ & 10.211 & 10.211 & 10.862 & 10.862 & 10.612 & & 11.170 & & 10.630 & \\
\hline & $4 \mathrm{~S}$ & $\overline{2}$ & 10.417 & 10.418 & 11.088 & 11.090 & & & & & 10.812 & \\
\hline & $5 S$ & & 10.606 & 10.610 & 11.297 & 11.301 & & & & & & \\
\hline & $2 S$ & & 9.990 & 9.988 & 10.619 & 10.617 & 10.592 & 10.578 & 10.770 & 10.501 & 10.482 & 10.574 \\
\hline & $3 S$ & $3^{+}$ & 10.205 & 10.233 & 10.855 & 10.866 & 10.593 & & 11.184 & & 10.673 & \\
\hline & $4 \mathrm{~S}$ & $\overline{2}$ & 10.418 & 10.420 & 11.090 & 11.092 & & & & & 10.856 & \\
\hline \multirow{9}{*}{$\begin{array}{l}\Xi_{b c d} \\
\text { and } \\
\Xi_{b c u}\end{array}$} & $5 S$ & & 10.607 & 10.611 & 11.298 & 11.302 & & & & & & \\
\hline & $2 S$ & & 6.922 & 6.919 & 7.244 & 7.240 & & & 7.478 & & & 7.321 \\
\hline & $3 S$ & $\underline{1}^{+}$ & 7.163 & 7.161 & 7.509 & 7.507 & & & 7.904 & & & \\
\hline & $4 \mathrm{~S}$ & $\overline{2}$ & 7.379 & 7.377 & 7.746 & 7.744 & & & & & & \\
\hline & $5 S$ & & 7.576 & 7.581 & 7.963 & 7.964 & & & & & & \\
\hline & $2 S$ & & 6.943 & 6.939 & 7.267 & 7.263 & & & 7.495 & & & 7.353 \\
\hline & $3 S$ & $3^{+}$ & 7.174 & 7.171 & 7.521 & 7.518 & & & 7.917 & & & \\
\hline & $4 \mathrm{~S}$ & $\overline{2}$ & 7.384 & 7.384 & 7.752 & 7.752 & & & & & & \\
\hline & $5 S$ & & 7.580 & 7.581 & 7.968 & 7.969 & & & & & & \\
\hline
\end{tabular}

$2 m_{\rho} m_{\lambda} /\left(m_{\rho}+m_{\lambda}\right)$ [32]. By regarding $\psi_{\nu \gamma}(x)=x^{-5 / 2} \varphi_{\nu \gamma}$ $[20,35],(11)$ reduces to the following form:

$$
\begin{gathered}
\varphi_{\nu \gamma}^{\prime \prime}(x)+\left[\varepsilon-r_{1} x^{2}-r_{2} x-\frac{r_{3}}{x}-\frac{r_{4}}{x^{2}}-\frac{r_{5}}{x^{3}}+\frac{r_{6}}{x^{5}}+r_{7}\right. \\
\left.-\frac{(2 \gamma+3)(2 \gamma+5)}{4 x^{2}}\right] \varphi_{\nu \gamma}(x)=0
\end{gathered}
$$

The hyperradial wave function $\varphi_{\nu \gamma}(x)$ is a solution of the reduced Schrödinger equation for each of the three identical particles with the mass $\mathrm{m}$ and interacting potential (8), where

$$
\begin{aligned}
& \varepsilon=2 m E, \\
& r_{1}=2 m p, \\
& r_{2}=2 m \beta, \\
& r_{3}=2 m \tau, \\
& r_{4}=2 m\left(\frac{1}{m_{\rho}}+\frac{1}{m_{\lambda}}\right)\left(-C_{f} C_{A} \frac{\alpha_{s}^{2}}{4}\right),
\end{aligned}
$$

$$
\begin{aligned}
r_{5} & =2 m\left[\frac{2 \tau}{3 m_{\rho} m_{\lambda}}\left(s_{\rho} \cdot S_{\lambda}\right)-\frac{3 \tau}{2 m_{\rho} m_{\lambda}}(\vec{\gamma} \cdot \vec{s})\right. \\
& \left.+\frac{7 \tau}{6 m_{\rho} m_{\lambda}} s^{2}\right], \\
r_{6} & =2 m \frac{21 \tau}{6 m_{\rho} m_{\lambda}}(\vec{s} \cdot \vec{x})(\vec{s} \cdot \vec{x}), \\
r_{7} & =2 m\left(\frac{(\beta+2 p)}{2 m_{\rho} m_{\lambda}}(\vec{\gamma} \cdot \vec{s})\right) .
\end{aligned}
$$

We suppose the $\varphi_{\nu \gamma}=h(x) e^{g(x)}$ form for the wave function. Now we make use of the ansatz for $h(x)$ and $g(x)$ $[33,34]$ :

$$
\begin{aligned}
& h(x)=\Pi\left(x-a_{i}^{\nu}\right) \quad v=1,2, \ldots, \\
& h(x)=1 \quad \nu=0 \\
& g(x)=a \ln x+q x^{2}+c x+\frac{d}{x}
\end{aligned}
$$


TABLE 4: The masses of orbital excited states for $\Xi_{c c}$ baryon (in $\mathrm{GeV}$ ). Standard devotions of the result are 0.072 and 0.068 .

(a)

\begin{tabular}{|c|c|c|c|c|c|c|c|c|c|c|c|c|}
\hline State & $\begin{array}{c}\text { Our cal } \\
\Xi_{c c}^{+}\end{array}$ & $\begin{array}{c}\text { Our Cal } \\
\Xi_{c c}^{++} \\
\end{array}$ & $\begin{array}{c}1] \\
\Xi_{c c}^{+} \\
\end{array}$ & $\begin{array}{l}{[1]} \\
\Xi_{c c}^{++} \\
\end{array}$ & [7] & {$[26]$} & [19] & {$[20]$} & [12] & {$[21]$} & {$[18]$} & {$[5]$} \\
\hline$\left(1^{2} P_{1 / 2}\right)$ & 3.851 & 3.847 & 3.865 & 3.861 & 3.947 & 3.910 & 3.880 & 3.838 & & & 4.073 & 3.892 \\
\hline$\left(1^{2} P_{3 / 2}\right)$ & 3.834 & 3.830 & 3.847 & 3.842 & 3.949 & 3.921 & & 3.959 & 3.786 & 3.834 & 4.079 & 3.989 \\
\hline$\left(1^{4} P_{1 / 2}\right)$ & 3.860 & 3.856 & 3.875 & 3.871 & & & & & & & & \\
\hline$\left(1^{4} P_{3 / 2}\right)$ & 3.842 & 3.838 & 3.856 & 3.851 & & & & & & & & \\
\hline$\left(1^{4} P_{5 / 2}\right)$ & 3.873 & 3.872 & 3.890 & 3.888 & 4.163 & 4.092 & & 4.155 & 3.949 & 4.047 & 4.089 & \\
\hline$\left(2^{2} P_{1 / 2}\right)$ & 4.120 & 4.101 & 4.161 & 4.140 & 4.135 & 4.074 & 4.018 & 4.085 & & & & \\
\hline$\left(2^{2} P_{3 / 2}\right)$ & 4.104 & 4.101 & 4.144 & 4.140 & 4.137 & 4.078 & 4.197 & & & & & \\
\hline$\left(2^{4} P_{1 / 2}\right)$ & 4.127 & 4.125 & 4.169 & 4.167 & & & & & & & & \\
\hline$\left(2^{4} P_{3 / 2}\right)$ & 4.111 & 4.109 & 4.152 & 4.149 & & & & & & & & \\
\hline$\left(2^{4} P_{5 / 2}\right)$ & 4.140 & 4.138 & 4.183 & 4.181 & 4.488 & & & & & & & \\
\hline$\left(3^{2} P_{1 / 2}\right)$ & 4.361 & 4.345 & 4.426 & 4.409 & 4.149 & & & & & & & \\
\hline$\left(3^{2} P_{3 / 2}\right)$ & 4.347 & 4.345 & 4.411 & 4.409 & 4.159 & & & & & & & \\
\hline$\left(3^{4} P_{1 / 2}\right)$ & 4.367 & 4.366 & 4.433 & 4.432 & & & & & & & & \\
\hline$\left(3^{4} P_{3 / 2}\right)$ & 4.354 & 4.352 & 4.419 & 4.417 & & & & & & & & \\
\hline$\left(3^{4} P_{5 / 2}\right)$ & 4.336 & 4.333 & 4.399 & 4.396 & 4.534 & & & & & & & \\
\hline$\left(4^{2} P_{1 / 2}\right)$ & 4.583 & 4.583 & 4.671 & 4.671 & & & & & & & & \\
\hline$\left(4^{2} P_{3 / 2}\right)$ & 4.571 & 4.571 & 4.658 & 4.657 & & & & & & & & \\
\hline$\left(4^{4} P_{1 / 2}\right)$ & 4.590 & 4.590 & 4.678 & 4.678 & & & & & & & & \\
\hline$\left(\begin{array}{ll}4^{4} & P_{3 / 2}\end{array}\right)$ & 4.577 & 4.577 & 4.664 & 4.664 & & & & & & & & \\
\hline$\left(4^{4} P_{5 / 2}\right)$ & 4.561 & 4.561 & 4.646 & 4.646 & & & & & & & & \\
\hline$\left(5^{2} P_{1 / 2}\right)$ & 4.792 & 4.793 & 4.901 & 4.902 & & & & & & & & \\
\hline$\left(\begin{array}{ll}5^{2} & P_{3 / 2}\end{array}\right)$ & 4.781 & 4.781 & 4.889 & 4.889 & & & & & & & & \\
\hline$\left(5^{4} P_{1 / 2}\right)$ & 4.799 & 4.800 & 4.908 & 4.909 & & & & & & & & \\
\hline$\left(5^{4} P_{3 / 2}\right)$ & 4.705 & 4.788 & 4.895 & 4.896 & & & & & & & & \\
\hline$\left(5^{4} P_{5 / 2}\right)$ & 4.771 & 4.772 & 4.878 & 4.879 & & & & & & & & \\
\hline$\left(1^{4} D_{1 / 2}\right)$ & 4.043 & 4.038 & 4.077 & 4.071 & & & & & & & & \\
\hline$\left(1^{2} D_{3 / 2}\right)$ & 4.018 & 4.013 & 4.049 & 4.044 & & & & & & & & \\
\hline$\left(\begin{array}{ll}1^{4} & D_{3 / 2}\end{array}\right)$ & 4.026 & 4.022 & 4.058 & 4.053 & & & & & & & & \\
\hline$\left(1^{2} D_{5 / 2}\right)$ & 3.995 & 3.991 & 4.024 & 4.019 & 4.043 & 4.115 & 4.047 & & 4.391 & 4.034 & 4.050 & 4.388 \\
\hline$\left(\begin{array}{ll}1^{4} & D_{5 / 2}\end{array}\right)$ & 4.003 & 4.000 & 4.033 & 4.029 & 4.027 & 4.052 & & 4.187 & 4.089 & 4.393 & & \\
\hline$\left(1^{4} D_{7 / 2}\right)$ & 3.975 & 3.972 & 4.002 & 3.998 & 4.097 & & & & & & & \\
\hline$\left(\begin{array}{ll}2^{4} & D_{1 / 2}\end{array}\right)$ & 4.287 & 4.284 & 4.345 & 4.342 & & & & & & & & \\
\hline$\left(2^{2} D_{3 / 2}\right)$ & 4.265 & 4.262 & 4.321 & 4.318 & & & & & & & & \\
\hline$\left(2^{4} D_{3 / 2}\right)$ & 4.272 & 4.270 & 4.329 & 4.326 & & & & & & & & \\
\hline$\left(2^{2} D_{5 / 2}\right)$ & 4.245 & 4.243 & 4.299 & 4.297 & 4.164 & 4.091 & & & & & & \\
\hline$\left(\begin{array}{ll}2^{4} & D_{5 / 2}\end{array}\right)$ & 4.252 & 4.251 & 4.307 & 4.305 & & & & & & & & \\
\hline$\left(\begin{array}{ll}2^{4} & D_{7 / 2}\end{array}\right)$ & 4.228 & 4.226 & 4.280 & 4.278 & 4.394 & & & & & & & \\
\hline \multicolumn{13}{|c|}{ (b) } \\
\hline State & $\begin{array}{c}\text { Our Cal } \\
\Xi_{c c}^{+}\end{array}$ & $\begin{array}{c}\text { Our Cal } \\
\Xi_{c c}^{++} \\
\end{array}$ & $\begin{array}{l}{[1]} \\
\Xi_{c c}^{+}\end{array}$ & $\begin{array}{l}1] \\
\Xi_{c c}^{++} \\
\end{array}$ & [7] & {$[32]$} & [33] & {$[34]$} & {$[35]$} & [33] & {$[36]$} & {$[5]$} \\
\hline$\left(3^{4} D_{1 / 2}\right)$ & 4.511 & 4.511 & 4.592 & 4.592 & 4.511 & 4.511 & 4.592 & 4.592 & 4.511 & 4.511 & 4.592 & 4.592 \\
\hline$\left(3^{2} D_{3 / 2}\right)$ & 4.492 & 4.491 & 4.571 & 4.570 & 4.492 & 4.491 & 4.571 & 4.570 & 4.492 & 4.491 & 4.571 & 4.570 \\
\hline$\left(\begin{array}{ll}3^{4} & D_{3 / 2}\end{array}\right)$ & 4.499 & 4.499 & 4.578 & 4.578 & & & & & & & & \\
\hline$\left(3^{2} D_{5 / 2}\right)$ & 4.475 & 4.474 & 4.552 & 4.551 & 4.348 & & & & & & & \\
\hline$\left(3^{4} D_{5 / 2}\right)$ & 4.481 & 4.481 & 4.559 & 4.558 & & & & & & & & \\
\hline$\left(3^{4} D_{7 / 2}\right)$ & 4.460 & 4.459 & 4.535 & 4.534 & & & & & & & & \\
\hline$\left(4^{4} D_{1 / 2}\right)$ & 4.723 & 4.724 & 4.825 & 4.826 & & & & & & & & \\
\hline$\left(\begin{array}{ll}4^{2} & D_{3 / 2}\end{array}\right)$ & 4.706 & 4.706 & 4.806 & 4.806 & & & & & & & & \\
\hline$\left(\begin{array}{ll}4^{4} & D_{3 / 2}\end{array}\right)$ & 4.711 & 4.712 & 4.812 & 4.813 & & & & & & & & \\
\hline
\end{tabular}


(b) Continued.

\begin{tabular}{|c|c|c|c|c|c|c|c|c|c|c|c|c|}
\hline State & $\begin{array}{c}\text { Our Cal } \\
\Xi_{c c}^{+}\end{array}$ & $\begin{array}{c}\text { Our Cal } \\
\Xi_{c c}^{++}\end{array}$ & $\begin{array}{l}{[1]} \\
\Xi_{c c}^{+} \\
\end{array}$ & $\begin{array}{l}{[1]} \\
\Xi_{c c}^{++} \\
\end{array}$ & [7] & [32] & {$[33]$} & {$[34]$} & {$[35]$} & {$[33]$} & {$[36]$} & {$[5]$} \\
\hline$\left(4^{2} D_{5 / 2}\right)$ & 4.690 & 4.690 & 4.788 & 4.788 & & & & & & & & \\
\hline$\left(4^{4} D_{5 / 2}\right)$ & 4.696 & 4.696 & 4.795 & 4.795 & & & & & & & & \\
\hline$\left(\begin{array}{ll}4^{4} & D_{7 / 2}\end{array}\right)$ & 4.675 & 4.675 & 4.772 & 4.772 & & & & & & & & \\
\hline$\left(1^{4} F_{3 / 2}\right)$ & 4.198 & 4.193 & 4.247 & 4.242 & & & & & & & & \\
\hline$\left(1^{2} F_{5 / 2}\right)$ & 4.169 & 4.164 & 4.215 & 4.210 & & & & & & & & \\
\hline$\left(\begin{array}{ll}1^{4} & F_{5 / 2}\end{array}\right)$ & 4.142 & 4.172 & 4.186 & 4.219 & & & & & & & & \\
\hline$\left(\begin{array}{ll}1^{4} & F_{7 / 2}\end{array}\right)$ & 4.150 & 4.147 & 4.194 & 4.191 & & & & & & & & \\
\hline$\left(1^{2} F_{7 / 2}\right)$ & 4.178 & 4.139 & 4.225 & 4.182 & & & & & 4.267 & & & \\
\hline$\left(1^{4} F_{9 / 2}\right)$ & 4.118 & 4.115 & 4.159 & 4.156 & & & & & 4.413 & & & \\
\hline$\left(2^{4} F_{3 / 2}\right)$ & 4.422 & 4.425 & 4.494 & 4.497 & & & & & & & & \\
\hline$\left(\begin{array}{ll}2^{2} & F_{5 / 2}\end{array}\right)$ & 4.399 & 4.399 & 4.468 & 4.468 & & & & & & & & \\
\hline$\left(2^{4} F_{5 / 2}\right)$ & 4.405 & 4.406 & 4.475 & 4.476 & & & & & & & & \\
\hline$\left(\begin{array}{ll}2^{4} & F_{7 / 2}\end{array}\right)$ & 4.378 & 4.382 & 4.445 & 4.450 & & & & & & & & \\
\hline$\left(2^{2} F_{7 / 2}\right)$ & 4.384 & 4.376 & 4.452 & 4.443 & & & & & & & & \\
\hline$\left(2^{4} F_{9 / 2}\right)$ & 4.359 & 4.355 & 4.424 & 4.420 & & & & & & & & \\
\hline
\end{tabular}

where $a, q, c$, and $d$ are positive. From (14), we obtain

$$
\begin{aligned}
& \varphi^{\prime \prime}(x) \\
& \quad=\left[g^{\prime \prime}(x)+g^{\prime 2}(x)+\left(\frac{h^{\prime \prime}(x)+2 h^{\prime}(x) g^{\prime}(x)}{h(x)}\right)\right] \\
& \quad \varphi(x)
\end{aligned}
$$

Comparing (12) and (15), it can be found that

$$
\begin{aligned}
& {\left[r_{1} x^{2}+r_{2} x+\frac{r_{3}}{x}+\frac{r_{4}}{x^{2}}+\frac{r_{5}}{x^{3}}-\frac{r_{6}}{x^{5}}-r_{7}\right.} \\
& \left.+\frac{(2 \gamma+3)(2 \gamma+5)}{4 x^{2}}-\varepsilon\right]=\left[g^{\prime \prime}(x)+g^{\prime 2}(x)\right. \\
& \left.+\frac{h^{\prime \prime}(x)+2 h^{\prime}(x) g^{\prime}(x)}{h(x)}\right]
\end{aligned}
$$

By substituting (14) into (16), we obtained the following equation:

$$
\begin{aligned}
-\varepsilon+ & r_{1} x^{2}+r_{2} x+\frac{r_{3}}{x}+\frac{r_{4}}{x^{2}}+\frac{r_{5}}{x^{3}}-\frac{r_{6}}{x^{5}}-r_{7} \\
& +\frac{(2 \gamma+3)(2 \gamma+5)}{4 x^{2}} \\
= & 4 q^{2} x^{2}+4 c q x+\frac{(2 a c-4 d q)}{x}+\frac{\left(a^{2}-a-2 c d\right)}{x^{2}} \\
& +\frac{2 d(1-a)}{x^{3}}+\frac{d^{2}}{x^{4}}+\left(c^{2}+2 q+4 a c\right)
\end{aligned}
$$

By equating the corresponding powers of $x$ on both sides of (17), we can obtain

$$
\begin{aligned}
a= & \frac{2 \tau}{\beta} \sqrt{\frac{m p}{2}}, \\
c= & \frac{m \beta}{2} \sqrt{\frac{2}{m p}}, \\
q= & \sqrt{\frac{m p}{2}}, \\
\varepsilon= & -\left[\frac{m \beta^{2}}{2 p}+2 \sqrt{\frac{m p}{2}}+\frac{4 m p \tau}{\beta}\right. \\
& \left.+2 m\left(\frac{(\beta+2 p)}{2 m_{\rho} m_{\lambda}}(\vec{\gamma} \cdot \vec{s})\right)\right]
\end{aligned}
$$

Since $p=m \omega^{2} / 2$, we have $a=2 m \omega / 2 \beta, c=\beta / \omega, q=m \omega / 2$. The energy eigenvalues for the mode $\nu=0$ and grand angular momentum $\gamma$ from (13) and (18) are given as follows:

$$
\begin{aligned}
E & =-\left[\frac{\beta^{2}}{2 m \omega}+\frac{\omega}{2}+\frac{m \omega^{2} \tau}{\beta}\right. \\
& \left.+\left(\frac{\left(\beta+m \omega^{2}\right)}{2 m_{\rho} m_{\lambda}}(\vec{\gamma} \cdot \vec{s})\right)\right]
\end{aligned}
$$

At last for the best doubly heavy baryons masses $\left(\Xi_{c c d}, \Xi_{c c u}\right.$, $\left.\Xi_{b b d}, \Xi_{b b u}, \Xi_{b c d}, \Xi_{b c u}\right)$ predictions, the values of $m_{u}, m_{d}, m_{c}$, $m_{b}, \alpha_{S}, \omega$, and $\beta$ (which are listed in Table 1 ) are selected using genetic algorithm. The cost function of a genetic algorithm is the minimum difference between our calculated baryon mass and the reported baryons mass of other works. 
TABLE 5: The masses of orbital excited states for $\Xi_{b b}$ baryon (in $\mathrm{GeV}$ ).

\begin{tabular}{|c|c|c|c|c|c|c|c|c|c|c|c|}
\hline State & $\begin{array}{c}\text { Our cal } \\
\Xi_{b b}^{-}\end{array}$ & $\begin{array}{c}\text { Our Cal } \\
\Xi_{b b}^{0}\end{array}$ & $\begin{array}{l}{[1]} \\
\Xi_{b b}^{-}\end{array}$ & $\begin{array}{l}{[1]} \\
\Xi_{b b}^{0}\end{array}$ & [7] & [26] & [19] & {$[20]$} & {$[12]$} & {$[18]$} & Others \\
\hline$\left(1^{2} P_{1 / 2}\right)$ & 9.895 & 9.892 & 10.514 & 10.511 & 10.476 & 10.493 & 10.406 & 10.368 & & 10.691 & \\
\hline$\left(1^{2} P_{3 / 2}\right)$ & 9.890 & 9.887 & 10.509 & 10.506 & 10.476 & 10.495 & & 10.408 & 10.474 & 10.692 & $10.390[31]$ \\
\hline$\left(\begin{array}{ll}1^{4} & P_{1 / 2}\end{array}\right)$ & 9.897 & 9.895 & 10.517 & 10.514 & & & & & & & \\
\hline$\left(1^{4} P_{3 / 2}\right)$ & 9.893 & 9.890 & 10.512 & 10.509 & & & & & & & $10.430[17]$ \\
\hline$\left(1^{4} P_{5 / 2}\right)$ & 9.901 & 9.898 & 10.521 & 10.518 & 10.759 & & & & 10.588 & 10.695 & \\
\hline$\left(2^{2} P_{1 / 2}\right)$ & 10.127 & 10.127 & 10.77 & 10.77 & 10.703 & 10.710 & 10612 & 10.563 & & & \\
\hline$\left(2^{2} P_{3 / 2}\right)$ & 10.124 & 10.120 & 10.766 & 10.762 & 10.704 & 10.713 & & 10.607 & & & \\
\hline$\left(2^{4} P_{1 / 2}\right)$ & 10.129 & 10.129 & 10.772 & 10.772 & & & & & & & \\
\hline$\left(2^{4} P_{3 / 2}\right)$ & 10.126 & 10.125 & 10.768 & 10.767 & & & & & & & \\
\hline$\left(2^{4} P_{5 / 2}\right)$ & 10.121 & 10.133 & 10.763 & 10.776 & 10.973 & 10.713 & & & & & \\
\hline$\left(3^{2} P_{1 / 2}\right)$ & 10.337 & 10.338 & 11.001 & 11.002 & 10.740 & & & 10.744 & & & \\
\hline$\left(3^{2} P_{3 / 2}\right)$ & 10.334 & 10.335 & 10.997 & 10.998 & 10.742 & & & 10.788 & & & \\
\hline$\left(3^{4} P_{1 / 2}\right)$ & 10.339 & 10.340 & 11.003 & 11.004 & & & & & & & \\
\hline$\left(3^{4} P_{3 / 2}\right)$ & 10.336 & 10.337 & 10.999 & 11.000 & & & & & & & \\
\hline$\left(3^{4} P_{5 / 2}\right)$ & 10.331 & 10.343 & 10.994 & 11.007 & 11.004 & & & & & & \\
\hline$\left(4^{2} P_{1 / 2}\right)$ & 10.531 & 10.534 & 11.214 & 11.217 & & & & 10.900 & & & \\
\hline$\left(4^{2} P_{3 / 2}\right)$ & 10.527 & 10.530 & 11.21 & 11.213 & & & & & & & \\
\hline$\left(4^{4} P_{1 / 2}\right)$ & 10.533 & 10.536 & 11.216 & 11.219 & & & & & & & \\
\hline$\left(4^{4} P_{3 / 2}\right)$ & 10.529 & 10.532 & 11.212 & 11.215 & & & & & & & \\
\hline$\left(4^{4} P_{5 / 2}\right)$ & 10.526 & 10.538 & 11.208 & 11.222 & & & & & & & \\
\hline$\left(5^{2} P_{1 / 2}\right)$ & 10.712 & 10.716 & 11.413 & 11.418 & & & & & & & \\
\hline$\left(5^{2} P_{3 / 2}\right)$ & 10.709 & 10.714 & 11.41 & 11.415 & & & & & & & \\
\hline$\left(5^{4} P_{1 / 2}\right)$ & 10.714 & 10.718 & 11.415 & 11.420 & & & & & & & \\
\hline$\left(5^{4} P_{3 / 2}\right)$ & 10.711 & 10.716 & 11.412 & 11.417 & & & & & & & \\
\hline$\left(5^{4} P_{5 / 2}\right)$ & 10.706 & 10.721 & 11.407 & 11.423 & & & & & & & \\
\hline$\left(\begin{array}{ll}1^{4} & D_{1 / 2}\end{array}\right)$ & 10.043 & 10.041 & 10.677 & 10.675 & & & & & & & \\
\hline$\left(\begin{array}{ll}1^{2} & D_{3 / 2}\end{array}\right)$ & 10.037 & 10.035 & 10.670 & 10.668 & & & & & & & \\
\hline$\left(\begin{array}{ll}1^{4} & D_{3 / 2}\end{array}\right)$ & 10.038 & 10.037 & 10.672 & 10.670 & & & & & & 11.011 & \\
\hline$\left(1^{2} D_{5 / 2}\right)$ & 10.030 & 10.028 & 10.663 & 10.661 & 10.592 & 10.676 & & & 10.742 & 11.002 & \\
\hline$\left(\begin{array}{ll}1^{4} & D_{5 / 2}\end{array}\right)$ & 10.033 & 10.031 & 10.666 & 10.664 & & & & & & & \\
\hline$\left(\begin{array}{ll}1^{4} & D_{7 / 2}\end{array}\right)$ & 10.026 & 10.024 & 10.658 & 10.656 & & 10.608 & & & 10.853 & 11.011 & \\
\hline$\left(2^{4} D_{1 / 2}\right)$ & 10.257 & 10.257 & 10.913 & 10.913 & & & & & & & \\
\hline$\left(2^{2} D_{3 / 2}\right)$ & 10.252 & 10.252 & 10.907 & 10.907 & & & & & & & \\
\hline$\left(2^{4} D_{3 / 2}\right)$ & 10.254 & 10.254 & 10.909 & 10.909 & & & & & & & \\
\hline$\left(\begin{array}{ll}2^{2} & D_{5 / 2}\end{array}\right)$ & 10.247 & 10.247 & 10.901 & 10.901 & & 10.712 & & & & & \\
\hline$\left(\begin{array}{ll}2^{4} & D_{5 / 2}\end{array}\right)$ & 10.248 & 10.248 & 10.903 & 10.903 & 10.613 & & & & & & \\
\hline$\left(\begin{array}{ll}2^{4} & D_{7 / 2}\end{array}\right)$ & 10.242 & 10.242 & 10.896 & 10.896 & & 11.057 & & & & & \\
\hline$\left(\begin{array}{ll}3^{4} & D_{1 / 2}\end{array}\right)$ & 10.455 & 10.457 & 11.13 & 11.133 & & & 4.592 & 4.592 & & & \\
\hline$\left(3^{2} D_{3 / 2}\right)$ & 10.450 & 10.452 & 11.125 & 11.127 & & & 4.571 & 4.570 & & & \\
\hline$\left(3^{4} D_{3 / 2}\right)$ & 10.451 & 10.454 & 11.126 & 11.129 & & & & & & & \\
\hline$\left(3^{2} D_{5 / 2}\right)$ & 10.446 & 10.447 & 11.120 & 11.122 & & & & & & & \\
\hline$\left(3^{4} D_{5 / 2}\right)$ & 10.447 & 10.449 & 11.122 & 11.124 & 10.809 & & & & & & \\
\hline$\left(3^{4} D_{7 / 2}\right)$ & 10.442 & 10.444 & 11.116 & 11.118 & & & & & & & \\
\hline$\left(4^{4} D_{1 / 2}\right)$ & 10.639 & 10.643 & 11.333 & 11.337 & & & & & & & \\
\hline$\left(4^{2} D_{3 / 2}\right)$ & 10.635 & 10.638 & 11.328 & 11.332 & & & & & & & \\
\hline$\left(\begin{array}{ll}4^{4} & D_{3 / 2}\end{array}\right)$ & 10.636 & 10.640 & 11.330 & 11.334 & & & & & & & \\
\hline$\left(4^{2} D_{5 / 2}\right)$ & 10.631 & 10.635 & 11.324 & 11.328 & & & & & & & \\
\hline
\end{tabular}


TABLE 5: Continued.

\begin{tabular}{|c|c|c|c|c|c|c|c|c|c|c|c|}
\hline State & $\begin{array}{c}\text { Our cal } \\
\Xi_{b b}^{-}\end{array}$ & $\begin{array}{c}\text { Our Cal } \\
\Xi_{b b}^{0}\end{array}$ & $\begin{array}{l}{[1]} \\
\Xi_{b b}^{-}\end{array}$ & $\begin{array}{l}{[1]} \\
\Xi_{b b}^{0}\end{array}$ & [7] & [26] & [19] & {$[20]$} & {$[12]$} & {$[18]$} & Others \\
\hline$\left(\begin{array}{ll}4^{4} & D_{5 / 2}\end{array}\right)$ & 10.632 & 10.636 & 11.325 & 11.33 & & & & & & & \\
\hline$\left(\begin{array}{ll}4^{4} & D_{7 / 2}\end{array}\right)$ & 10.627 & 10.631 & 11.320 & 11.324 & & & & & & & \\
\hline$\left(1^{4} F_{3 / 2}\right)$ & 10.173 & 10.172 & 10.82 & 10.819 & & & & & & & \\
\hline$\left(1^{2} F_{5 / 2}\right)$ & 10.166 & 10.165 & 10.812 & 10.811 & & & & & & & \\
\hline$\left(1^{4} F_{5 / 2}\right)$ & 10.158 & 10.167 & 10.804 & 10.813 & & & & & & & \\
\hline$\left(1^{4} F_{7 / 2}\right)$ & 10.167 & 10.160 & 10.814 & 10.806 & & & & & & & \\
\hline$\left(1^{2} F_{7 / 2}\right)$ & 10.160 & 10.157 & 10.806 & 10.803 & & & & & 11.004 & & \\
\hline$\left(1^{4} F_{9 / 2}\right)$ & 10.152 & 10.152 & 10.797 & 10.797 & & & & & 11.112 & & \\
\hline$\left(2^{4} F_{3 / 2}\right)$ & 10.357 & 10.376 & 11.022 & 11.043 & & & & & & & \\
\hline$\left(2^{2} F_{5 / 2}\right)$ & 10.368 & 10.369 & 11.035 & 11.036 & & & & & & & \\
\hline$\left(2^{4} F_{5 / 2}\right)$ & 10.369 & 10.371 & 11.036 & 11.038 & & & & & & & \\
\hline$\left(\begin{array}{ll}2^{4} & F_{7 / 2}\end{array}\right)$ & 10.362 & 10.365 & 11.028 & 11.031 & & & & & & & \\
\hline$\left(2^{2} F_{7 / 2}\right)$ & 10.364 & 10.363 & 11.030 & 11.029 & & & & & & & \\
\hline$\left(2^{4} F_{9 / 2}\right)$ & 10.357 & 10.357 & 11.022 & 11.023 & & & & & & & \\
\hline
\end{tabular}

\section{Results and Discussions: Mass Spectrum}

The ground and excited states of doubly heavy $\Xi$ baryons are unclear to us experimentally (except $\Xi_{c c}^{+}$and $\Xi_{c c}^{++}$). Hence, we have obtained the ground and excited state masses of $\Xi_{c c}^{+}, \Xi_{c c}^{++}$, $\Xi_{b b}^{-}, \Xi_{b b}^{0}, \Xi_{b c}^{0}$, and $\Xi_{b c}^{+}$(see Tables $2,3,4,5$, and 6 , respectively). These mass spectra are estimated by using the hypercentral potential equation (8) in the hypercentral constituent quark model. We begin with the ground state $1 S$; the masses are computed for both parities $J^{P}=(1 / 2)^{+}$and $J^{P}=(3 / 2)^{+}$. Our predicted ground state masses of doubly heavy $\Xi$ baryons are compared with other predictions in Table 2.

We can observe that, in the case of $\Xi_{c c}$ baryon, for $2 S$ states $J^{P}=(1 / 2)^{+}$and $J^{P}=(3 / 2)^{+}$, our predictions are close to [34] and [1], respectively. Our outcomes for $3 \mathrm{~S}$ state $J^{P}=(1 / 2)^{+}$ of $\Xi_{c c}$ baryon show $21 \mathrm{MeV}$ (with [7]) and $J^{P}=(3 / 2)^{+}$shows $51 \mathrm{MeV}$ (with [1]) difference. Analyzing the $2 \mathrm{~S}$ and $3 \mathrm{~S}$ states masses for $\Xi_{b b}$ and $\Xi_{b c}$ baryons (with both parities) shows that our masses have a difference in the range of $\approx 0.5 \mathrm{GeV}$ with $[1,7,20,32-34,36]$.

To calculate the orbital excited state masses (1P-5P, $1 \mathrm{D}-4 \mathrm{D}, 1 \mathrm{~F}-2 \mathrm{~F})$, we have considered all possible isospin splitting and all combinations of total spin $S$ and total angular momentum J. Our outcomes and the comparison of masses with other approaches are also tabulated in Tables 4,5 , and 6.

Our obtained orbital excited masses for $\Xi_{c c}, 1 \mathrm{P}$ state $J^{P}=$ $(1 / 2)^{-}$show a difference of $14 \mathrm{MeV}$ (with [1]), $29 \mathrm{MeV}$ (with [33]), $13 \mathrm{MeV}$ (with [34]), and $41 \mathrm{MeV}$ (with [5]), while $1 P$ state $J^{P}=(3 / 2)^{-}$shows $14 \mathrm{MeV}$ (with [1]), $48 \mathrm{MeV}$ (with [35]), and $0 \mathrm{MeV}$ (with [33] ). Our 2P state $J^{P}=(1 / 2)^{-}$shows a difference of $15 \mathrm{MeV}$ (with [7]), $35 \mathrm{MeV}$ (with [34]), and $41 \mathrm{MeV}$ (with [1]), while $2 \mathrm{P}$ state $J^{P}=(3 / 2)^{-}$shows $26 \mathrm{MeV}$ (with [32]), $33 \mathrm{MeV}$ (with [7]), and $40 \mathrm{MeV}$ (with [1]). Results for $3 \mathrm{P}$ states $J^{P}=(1 / 2)^{-}$and $J^{P}=(3 / 2)^{-}$show a difference in the range of $\approx 60 \mathrm{MeV}$ with [1]. We can easily observe that our calculated masses for $4 \mathrm{P}-5 \mathrm{P}, 1 \mathrm{D}-3 \mathrm{D}$, and $1 \mathrm{~F}-2 \mathrm{~F}$ are matched with [1]. Our outcome for $3 \mathrm{D}$ state $J^{P}=(3 / 2)^{+}$is quite equal to the predictions of $[7,32,33,35]$. For the ground and excited states of doubly heavy baryons $\left(\Xi_{c c}^{+}\right)$, the minimum and maximum percentage of relative error values are $0 \%$ and $3.53 \%$ between our calculations and the masses reported by Shah et al. [1].

For $\Xi_{b b}$ and $\Xi_{b c}$ baryons, the mass difference from our calculations and other references is large.

Comparing our findings with the masses reported by Shah et al. [1], the minimum and maximum percentage of relative error values are $1.2 \%(0.8 \%)$ and $10.317 \%(6.92 \%)$ for the ground and excited states of doubly heavy baryons $\Xi_{b b}$ and $\Xi_{b c}$, respectively.

\section{Conclusion}

In this study, we have computed the mass spectra of ground and excited states for doubly heavy $\Xi$ baryons by using a hypercentral constituent quark model. For this goal, we have analytically solved the hyperradial Schrödinger equation for three identical interacting particles under the effective hypercentral potential by using the ansatz method. Our proposed potential is regarded as a combination of the Coulombic-like term plus a linear confining term and the harmonic oscillator potential. We also added the first-order correction and the spin-dependent part to the potential. In our calculations, the $u$ and $d$ quarks have $10 \mathrm{MeV}$ difference mass, so there is a very small mass difference between $\Xi_{c c d}$ and $\Xi_{c c u}, \Xi_{b b d}$ and $\Xi_{b b u}, \Xi_{b c d}$ and $\Xi_{b c u}$. Our model has succeeded to assign the $J^{P}$ values to the exited states of doubly heavy baryons $\left(\Xi_{c c d}, \Xi_{c c u}\right.$, $\Xi_{b b d}, \Xi_{b b u}, \Xi_{b c d}$, and $\left.\Xi_{b c u}\right)$. Comparison of the results with other predictions revealed that they are in agreement and our proposed model can be useful to investigate the doubly heavy baryons states masses. For example, for the ground, radial, and orbital excited states masses of doubly heavy $\Xi$ baryons the minimum and the maximum percentage of relative error values are $0 \%$ and $6 \%$ between our calculations and the masses reported by Shah et al. [1]. 
TABLE 6: The masses of orbital excited states for $\Xi_{b c}$ baryon (in $\mathrm{GeV}$ ).

\begin{tabular}{|c|c|c|c|c|c|}
\hline State & $\begin{array}{c}\text { Our cal } \\
\Xi_{b c}^{0}\end{array}$ & $\begin{array}{c}\text { Our Cal } \\
\Xi_{b c}^{+}\end{array}$ & $\begin{array}{l}1] \\
\Xi_{b c}^{0}\end{array}$ & $\begin{array}{l}{[1]} \\
\Xi_{b c}^{+}\end{array}$ & {$[18]$} \\
\hline$\left(1^{2} P_{1 / 2}\right)$ & 6.846 & 6.842 & 7.16 & 7.156 & 7.390 \\
\hline$\left(1^{2} P_{3 / 2}\right)$ & 6.836 & 6.831 & 7.149 & 7.144 & 7.394 \\
\hline$\left(1^{4} P_{1 / 2}\right)$ & 6.851 & 6.847 & 7.166 & 7.161 & 7.399 \\
\hline$\left(1^{4} P_{3 / 2}\right)$ & 6.841 & 6.837 & 7.155 & 7.15 & \\
\hline$\left(1^{4} P_{5 / 2}\right)$ & 6.859 & 6.856 & 7.175 & 7.171 & \\
\hline$\left(2^{2} P_{1 / 2}\right)$ & 7.087 & 7.084 & 7.425 & 7.422 & \\
\hline$\left(2^{2} P_{3 / 2}\right)$ & 7.078 & 7.075 & 7.415 & 7.412 & \\
\hline$\left(2^{4} P_{1 / 2}\right)$ & 7.091 & 7.088 & 7.43 & 7.426 & \\
\hline$\left(2^{4} P_{3 / 2}\right)$ & 7.082 & 7.079 & 7.42 & 7.417 & \\
\hline$\left(2^{4} P_{5 / 2}\right)$ & 7.071 & 7.095 & 7.408 & 7.434 & \\
\hline$\left(3^{2} P_{1 / 2}\right)$ & 7.304 & 7.302 & 7.664 & 7.662 & \\
\hline$\left(3^{2} P_{3 / 2}\right)$ & 7.296 & 7.295 & 7.655 & 7.654 & \\
\hline$\left(3^{4} P_{1 / 2}\right)$ & 7.308 & 7.306 & 7.668 & 7.666 & \\
\hline$\left(3^{4} P_{3 / 2}\right)$ & 7.299 & 7.299 & 7.659 & 7.658 & \\
\hline$\left(3^{4} P_{5 / 2}\right)$ & 7.289 & 7.312 & 7.648 & 7.673 & \\
\hline$\left(\begin{array}{ll}4^{2} & P_{1 / 2}\end{array}\right)$ & 7.504 & 7.623 & 7.884 & 8.015 & \\
\hline$\left(4^{2} P_{3 / 2}\right)$ & 7.497 & 7.498 & 7.876 & 7.877 & \\
\hline$\left(4^{4} P_{1 / 2}\right)$ & 7.508 & 7.508 & 7.888 & 7.888 & \\
\hline$\left(4^{4} P_{3 / 2}\right)$ & 7.500 & 7.500 & 7.88 & 7.88 & \\
\hline$\left(4^{4} P_{5 / 2}\right)$ & 7.491 & 7.514 & 7.87 & 7.895 & \\
\hline$\left(5^{2} P_{1 / 2}\right)$ & 7.692 & 7.693 & 8.091 & 8.092 & \\
\hline$\left(5^{2} P_{3 / 2}\right)$ & 7.686 & 7.687 & 8.084 & 8.085 & \\
\hline$\left(5^{4} P_{1 / 2}\right)$ & 7.695 & 7.697 & 8.094 & 8.096 & \\
\hline$\left(5^{4} P_{3 / 2}\right)$ & 7.689 & 7.689 & 8.087 & 8.088 & \\
\hline$\left(5^{4} P_{5 / 2}\right)$ & 7.680 & 7.681 & 8.078 & 8.079 & \\
\hline$\left(\begin{array}{ll}1^{4} & D_{1 / 2}\end{array}\right)$ & 7.006 & 7.004 & 7.336 & 7.334 & \\
\hline$\left(1^{2} D_{3 / 2}\right)$ & 6.992 & 6.989 & 7.321 & 7.318 & \\
\hline$\left(\begin{array}{ll}1^{4} & D_{3 / 2}\end{array}\right)$ & 6.997 & 6.980 & 7.326 & 7.308 & 7.324 \\
\hline$\left(1^{2} D_{5 / 2}\right)$ & 6.980 & 6.977 & 7.308 & 7.304 & \\
\hline$\left(\begin{array}{ll}1^{4} & D_{5 / 2}\end{array}\right)$ & 6.985 & 6.969 & 7.313 & 7.295 & 7.309 \\
\hline$\left(\begin{array}{ll}1^{4} & D_{7 / 2}\end{array}\right)$ & 6.969 & 6.953 & 7.296 & 7.278 & 7.292 \\
\hline$\left(2^{4} D_{1 / 2}\right)$ & 7.087 & 7.227 & 7.425 & 7.579 & 7.579 \\
\hline$\left(2^{2} D_{3 / 2}\right)$ & 7.216 & 7.214 & 7.567 & 7.565 & \\
\hline$\left(\begin{array}{ll}2^{4} & D_{3 / 2}\end{array}\right)$ & 7.219 & 7.219 & 7.571 & 7.57 & \\
\hline$\left(2^{2} D_{5 / 2}\right)$ & 7.205 & 7.203 & 7.555 & 7.553 & 7.538 \\
\hline$\left(2^{4} D_{5 / 2}\right)$ & 7.209 & 7.208 & 7.559 & 7.558 & \\
\hline$\left(\begin{array}{ll}2^{4} & D_{7 / 2}\end{array}\right)$ & 7.196 & 7.195 & 7.545 & 7.544 & \\
\hline$\left(3^{4} D_{1 / 2}\right)$ & 7.431 & 7.431 & 7.804 & 7.804 & \\
\hline$\left(3^{2} D_{3 / 2}\right)$ & 7.420 & 7.420 & 7.792 & 7.792 & \\
\hline$\left(3^{4} D_{3 / 2}\right)$ & 7.411 & 7.424 & 7.782 & 7.796 & \\
\hline$\left(3^{2} D_{5 / 2}\right)$ & 7.415 & 7.410 & 7.786 & 7.781 & \\
\hline$\left(3^{4} D_{5 / 2}\right)$ & 7.402 & 7.414 & 7.772 & 7.785 & \\
\hline$\left(3^{4} D_{7 / 2}\right)$ & 7.402 & 7.402 & 7.772 & 7.772 & \\
\hline$\left(4^{4} D_{1 / 2}\right)$ & 7.429 & 7.504 & 7.801 & 7.884 & 7.797 \\
\hline$\left(\begin{array}{ll}4^{2} & D_{3 / 2}\end{array}\right)$ & 7.611 & 7.613 & 8.002 & 8.004 & \\
\hline$\left(\begin{array}{ll}4^{4} & D_{3 / 2}\end{array}\right)$ & 7.615 & 7.617 & 8.006 & 8.008 & \\
\hline$\left(\begin{array}{ll}4^{2} & D_{5 / 2}\end{array}\right)$ & 7.603 & 7.604 & 7.993 & 7.994 & \\
\hline$\left(\begin{array}{ll}4^{4} & D_{5 / 2}\end{array}\right)$ & 7.606 & 7.608 & 7.996 & 7.998 & \\
\hline$\left(\begin{array}{ll}4^{4} & D_{7 / 2}\end{array}\right)$ & 7.596 & 7.597 & 7.985 & 7.986 & \\
\hline$\left(1^{4} F_{3 / 2}\right)$ & 7.143 & 7.141 & 7.487 & 7.485 & \\
\hline$\left(1^{2} F_{5 / 2}\right)$ & 7.127 & 7.125 & 7.469 & 7.467 & \\
\hline
\end{tabular}


TABLE 6: Continued.

\begin{tabular}{|c|c|c|c|c|c|}
\hline State & $\begin{array}{c}\text { Our cal } \\
\Xi_{b c}^{0}\end{array}$ & $\begin{array}{c}\text { Our Cal } \\
\Xi_{b c}^{+}\end{array}$ & $\begin{array}{l}{[1]} \\
\Xi_{b c}^{0}\end{array}$ & $\begin{array}{l}{[1]} \\
\Xi_{b c}^{+}\end{array}$ & [18] \\
\hline$\left(1^{4} F_{5 / 2}\right)$ & 7.131 & 7.129 & 7.474 & 7.472 & \\
\hline$\left(\begin{array}{ll}1^{4} & F_{7 / 2}\end{array}\right)$ & 7.117 & 7.114 & 7.458 & 7.455 & \\
\hline$\left(1^{2} F_{7 / 2}\right)$ & 7.112 & 7.109 & 7.453 & 7.45 & \\
\hline$\left(1^{4} F_{9 / 2}\right)$ & 7.099 & 7.097 & 7.439 & 7.436 & \\
\hline$\left(2^{4} F_{3 / 2}\right)$ & 7.350 & 7.350 & 7.715 & 7.715 & \\
\hline$\left(2^{2} F_{5 / 2}\right)$ & 7.337 & 7.336 & 7.7 & 7.699 & \\
\hline$\left(2^{4} F_{5 / 2}\right)$ & 7.340 & 7.339 & 7.704 & 7.703 & \\
\hline$\left(\begin{array}{ll}2^{4} & F_{7 / 2}\end{array}\right)$ & 7.328 & 7.327 & 7.69 & 7.689 & \\
\hline$\left(2^{2} F_{7 / 2}\right)$ & 7.324 & 7.323 & 7.686 & 7.685 & \\
\hline$\left(\begin{array}{ll}2^{4} & F_{9 / 2}\end{array}\right)$ & 7.313 & 7.311 & 7.674 & 7.672 & \\
\hline
\end{tabular}

\section{Data Availability}

The data used to support the findings of this study are included within the article.

\section{Conflicts of Interest}

The authors declare that they have no conflicts of interest.

\section{References}

[1] Z. Shah and A. K. Rai, "Excited state mass spectra of doubly heavy $\Xi$ baryons," The European Physical Journal C, vol. 77, no. 2, 2017.

[2] SELEX Collaboration, A. Ocherashvili, M. A. Moinester, J. Russ et al., "Confirmation of the doubly charmed baryon $\Xi_{c c}^{+}(3520)$ via its decay to $p D^{+} \mathrm{K}^{-}$," Physics Letters $B$, vol. 628, article 18, 2005.

[3] K. A. Olive, K. Agashe, and C. Amsler, "Review of particle physics," Chinese Physics C, vol. 38, no. 9, Article ID 090001, 2014.

[4] S. Koshkarev and V. Anikeev, "Production of the doubly charmed baryons at the SELEX experiment - The double intrinsic charm approach," Physics Letters B, vol. 765, pp. 171174, 2017.

[5] P. P. Rubio, S. Collins, and G. S. Baliy, "Charmed baryon spectroscopy and light flavor symmetry from lattice QCD," Physical Review D, vol. 92, article 034504, 2015.

[6] R. M. Woloshyn and M. Wurtz, "Systematics of radial excitations in heavy-light hadrons," https://arxiv.org/abs/1601.01925.

[7] T. Yoshida, E. Hiyama, A. Hosaka, M. Oka, and K. Sadato, "Spectrum of heavy baryons in the quark model," Physical Review D: Particles, Fields, Gravitation and Cosmology, vol. 92, no. 11, 2015.

[8] D. Ebert, R. N. Faustov, and V. O. Galkin, "Spectroscopy and Regge trajectories of heavy baryons in the relativistic quarkdiquark picture," Physical Review D: Particles, Fields, Gravitation and Cosmology, vol. 84, no. 1, 2011.

[9] C. García-Recio, J. Nieves, O. Romanets, L. L. Salcedo, and L. Tolos, "Hidden charm $N$ and $\Delta$ resonances with heavy-quark symmetry," Physical Review D: Particles, Fields, Gravitation and Cosmology, vol. 87, no. 7, article 034032, 2013.
[10] Q. Mao, H. X. Chen, W. Chen et al., "QCD sum rule calculation for P-wave bottom baryons," Physical Review D, vol. 92, article 114007, 2015.

[11] Y. Yamaguchi, S. Ohkoda, A. Hosaka, T. Hyodo, and S. Yasui, "Heavy quark symmetry in multihadron systems," Physical Review D: Particles, Fields, Gravitation and Cosmology, vol. 91, no. 3, 2015.

[12] K. W. Wei, B. Chen, and X. H. Guo, "Masses of doubly and triply charmed baryons," Physical Review D, vol. 92, article 076008, 2015.

[13] K. W. Wei, B. Chen, N. Liu et al., "Spectroscopy of singly, doubly, and triply bottom baryons," Physical Review D, vol. 95, article 116005, 2016.

[14] Z. S. Brown, W. Detmold, S. Meinel, and K. Orginos, "Charmed bottom baryon spectroscopy from lattice QCD," Physical Review D, vol. 90, article 094507, Article ID 094507, 2014.

[15] M. Padmanath, R. G. Edwards, N. Mathur, and M. Peardon, "Spectroscopy of doubly charmed baryons from lattice QCD," Physical Review D: Particles, Fields, Gravitation and Cosmology, vol. 91, no. 9, 2015.

[16] T. M. Aliev, K. Azizi, and M. Savei, "Doubly heavy spin-1/2 baryon spectrum in QCD," Nuclear Physics A, vol. 895, article 59, 2012.

[17] T. M. Aliev, K. Azizi, and M. Savci, "The masses and residues of doubly heavy spin-3/2 baryons," Journal of Physics G: Nuclear and Particle Physics, vol. 40, article 065003, 2013.

[18] B. Eakins and W. Roberts, "Symmetries and Systematics of Doubly Heavy Hadrons," International Journal of Modern Physics A, vol. 27, article 1250039, 2012.

[19] A. Valcarce, H. Garcilazo, and J. Vijande, "Towards an understanding of heavy baryon spectroscopy," The European Physical Journal A, vol. 37, article 217, 2008.

[20] D. Ebert, R. N. Faustov, V. O. Galkin, and A. P. Martynenko, "Mass spectra of doubly heavy baryons in the relativistic quark model," Physical Review D: Particles, Fields, Gravitation and Cosmology, vol. 66, no. 1, 2002.

[21] S. S. Gershtein, V. V. Kiselev, A. K. Likhoded, and A. I. Onishchenko, "Spectroscopy of doubly heavy baryons," Physical Review D: Particles, Fields, Gravitation and Cosmology, vol. 62, no. 5, 2000.

[22] C. Albertus, E. Hernandez, J. Nieves, and J. M. Verde-Velasco, "Static properties and semileptonic decays of doubly heavy baryons in a nonrelativistic quark model," The European Physical Journal A, vol. 32, article 183, 2007. 
[23] N. Salehi, A. A. Rajabi, and Z. Ghalenovi, "Spectrum of strange and nonstrange baryons by using generalized gürsey radicati mass formula and hypercentral potential," Acta Physica Polonica, vol. 42, 2011.

[24] C. Patrignani and Particle Data Group, "Review of Particle Physics," Chinese Physics C, vol. 40, article 100001, 2016.

[25] C. Alexandrou, V. Drach, K. Jansen, C. Kallidonis, and G. Koutsou, "Baryon spectrum with ," Physical Review D: Particles, Fields, Gravitation and Cosmology, vol. 90, no. 7, 2014.

[26] W. Roberts and M. Pervin, "Heavy Baryons in a Quark Model," International Journal of Modern Physics A, vol. 23, article 2817, 2008.

[27] F. Giannuzzi, "Doubly heavy baryons in a Salpeter model with AdS/QCD inspired potential," Physical Review D: Particles, Fields, Gravitation and Cosmology, vol. 79, no. 9, 2009.

[28] M. Karliner and J. L. Rosner, "Baryons with two heavy quarks: Masses, production, decays, and detection," Physical Review D: Particles, Fields, Gravitation and Cosmology, vol. 90, no. 9, 2014.

[29] L. Tang, X.-H. Yuan, C.-F. Qiao, and X.-Q. Li, "Study of Doubly Heavy Baryon Spectrum via QCD Sum Rules," Communications in Theoretical Physics, vol. 57, article 435, 2012.

[30] A. P. Martynenko, "Ground-state triply and doubly heavy baryons in a relativistic three-quark model," Physics Letters B, vol. 663 , article 317, 2008.

[31] Z. G. Wang, "Analysis of the (1/2) - and (3/2)- heavy and doubly heavy baryon states with QCD sum rules," The European Physical Journal A, vol. 47, article 267, 2010.

[32] U. Loring, K. Kretzschmar, B. C. Metsch, and H. R. Petry, "Relativistic quark models of baryons with instantaneous forces," The European Physical Journal A, vol. 10, article 309, 2001.

[33] A. A. Rajabi and N. Salehi, "Mesons states and their dependence on spin and isospin," Iranian Journal of Physics Research, vol. 8, no. 3, pp. 169-175, 2008.

[34] N. Salehi, "A New Method for Obtaining the Baryons Mass under the Killingbeck Plus Isotonic Oscillator Potentials," Advances in High Energy Physics, vol. 2016, Article ID 5054620, 9 pages, 2016.

[35] Z. Shah, K. Thakkar, A. K. Rai et al., "Mass spectra and Regge trajectories of $\Lambda_{c}^{+}, \Sigma^{0} c, \Xi_{c}^{0}$ and $\Omega_{c}^{0}$ baryons ${ }^{\star}$," Chinese Physics $C$, vol. 40, article 123102, 2016.

[36] N. Salehi, H. Hassanabadi, and A. A. Rajabi, "The light and strange baryon spectrum in a non-relativistic hypercentral quark potential model and algebraic framework," The European Physical Journal Plus, vol. 128, p. 27, 2013.

[37] L. I. Abou-Salem, "Study of baryon spectroscopy using a new potential form," Advances in High Energy Physics, vol. 2014, Article ID 196484, 5 pages, 2014.

[38] Z. Shah, K. Thakkar, A. Kumar Rai, and P. C. Vinodkumar, "Excited state mass spectra of singly charmed baryons," The European Physical Journal A, vol. 52, article 313, 2016.

[39] H. Garcila, J. Vijande, and A. Valcarce, "Faddeev study of heavybaryon spectroscopy," Journal of Physics G: Nuclear and Particle Physics, vol. 34, article 961, 2007.

[40] E. Santopinto, M. M. Giannini, and F. Iachello, Symmetries in Science VII, B. Gruber, Ed., Plenum Press, New York, NY, USA, 1995.

[41] F. Iachello, Symmetries in Science VII, B. Gruber, Ed., Plenum Press, New York, NY, USA, 1995.

[42] L. Y. Glozman and D. O. Riska, "The spectrum of the nucleons and the strange hyperons and chiral dynamics," Physics Reports, vol. 268, article 263, 1996.
[43] K. Thakkar, Z. Shah, A. K. Rai et al., "Excited state mass spectra and Regge trajectories of bottom baryons," Nuclear Physics A, vol. 965, pp. 57-73, 2017.

[44] Y. Koma, M. Koma, and H. Wittig, “ Nonperturbative Determination of the QCD Potential at ," Physical Review Letters, vol. 97, no. 12,2006

[45] E. Santopinto, F. Iachello, and M. M. Giannini, "Nucleon form factors in a simple three-body quark model," The European Physical Journal A, vol. 1, p. 307, 1998.

[46] A. K. Rai and D. P. Rathaud, "The mass spectra and decay properties of dimesonic states, using the Hellmann potential," The European Physical Journal C, vol. 75, no. 9, 2015.

[47] H. Mariji, "Imposing Fermi momentum cut-off on the channeland density-dependent effective interaction and the groundstate properties of closed shell nuclei," The European Physical Journal A, vol. 50, no. 3, article 56, 2014.

[48] Z. Shah and A. K. Rai, "Masses and electromagnetic transitions of the $\mathrm{B}_{c}$ mesons," The European Physical Journal A, vol. 53, article 195, 2017.

[49] W. Lucha and F. F. Schoberl, "Solving the Schrodinger equation for bound states with Mathematica 3.0," International Journal of Modern Physics C, vol. 10, no. 4, pp. 607-619, 1999.

[50] J. Ballot and M. Fabre de la Ripelle, "Application of the hyperspherical formalism to the trinucleon bound state problems," Annals of Physics, vol. 127, article 62, 1980.

[51] Z. Shah, K. Thakkar, and A. K. Rai, "Excited state mass spectra of doubly heavy baryons $\Omega_{c c}, \Omega_{b b}$, and $\Omega_{b c}$," The European Physical Journal C, vol. 76, article 530, 2016. 

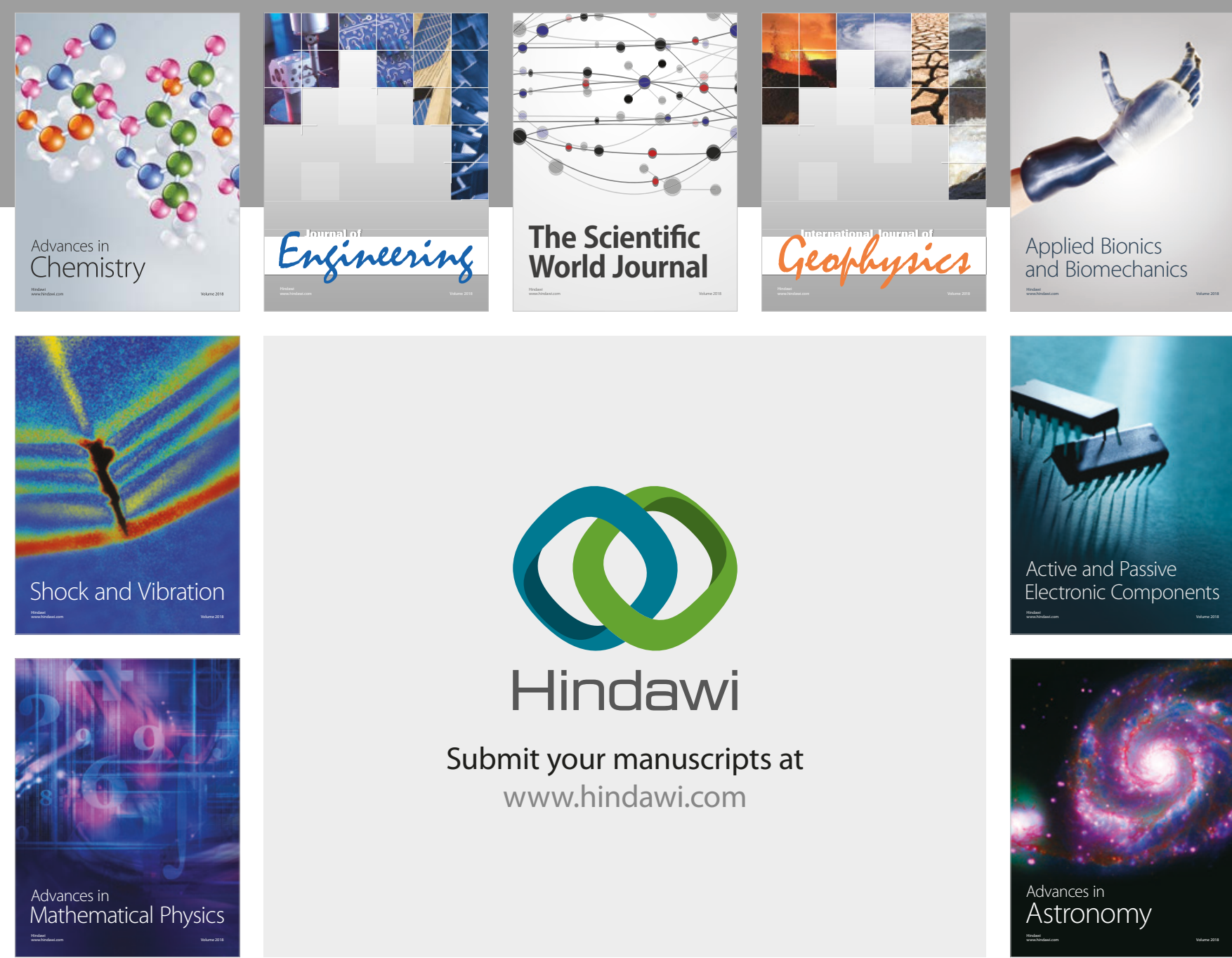

Submit your manuscripts at

www.hindawi.com

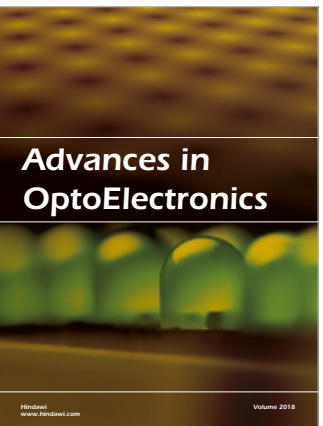

\section{Rotcting Machinery}
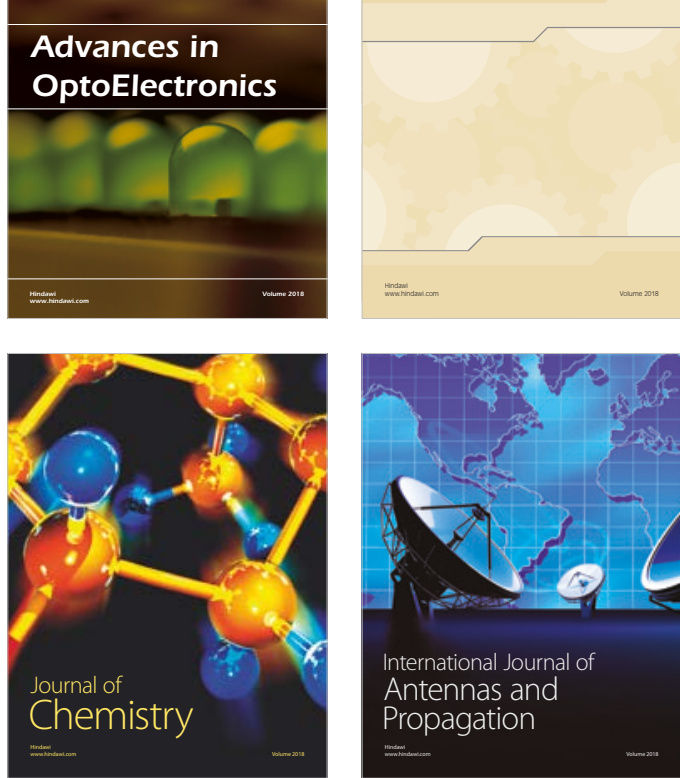

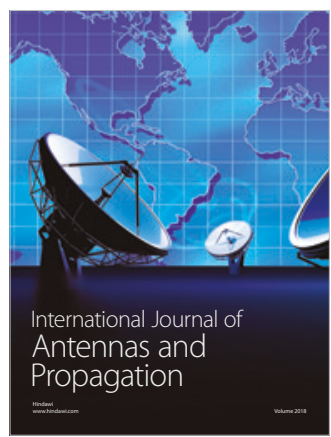

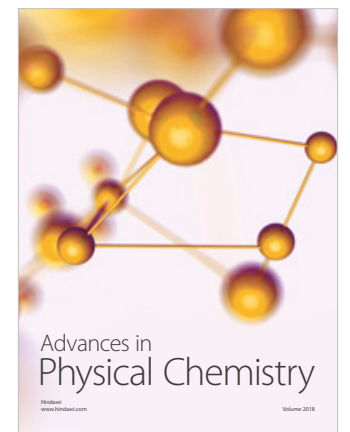

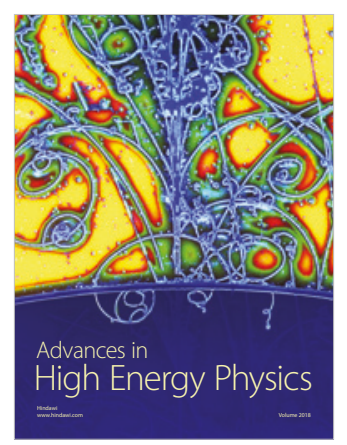

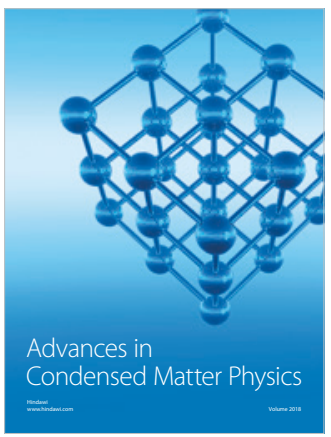

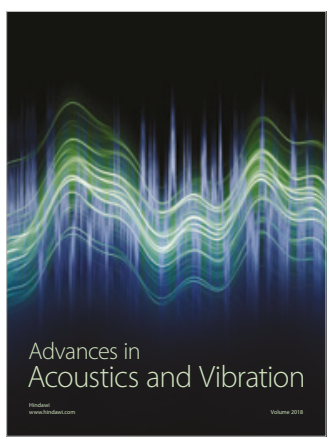

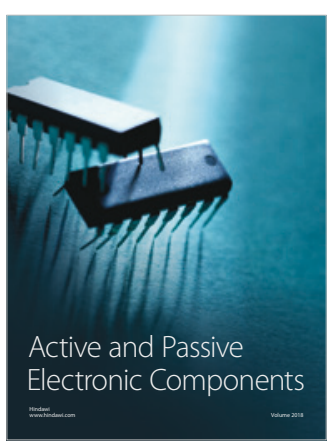
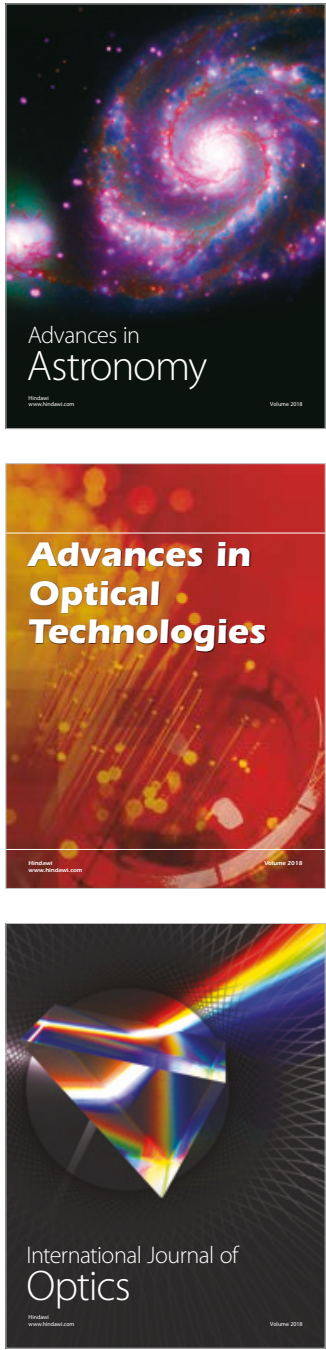